\title{
Ultrafast dynamic conductivity and scattering rate saturation of photoexcited charge carriers in silicon investigated with a midinfrared continuum probe
}

Fanqi Meng, Mark D. Thomson, Bo Sernelius, Michael Joerger and Hartmut G. Roskos

\author{
Linköping University Post Print
}

\section{Tweet}

N.B.: When citing this work, cite the original article.

Original Publication:

Fanqi Meng, Mark D. Thomson, Bo Sernelius, Michael Joerger and Hartmut G. Roskos, Ultrafast dynamic conductivity and scattering rate saturation of photoexcited charge carriers in silicon investigated with a midinfrared continuum probe, 2015, Physical Review B. Condensed Matter and Materials Physics, (91), 7, 075201.

http://dx.doi.org/10.1103/PhysRevB.91.075201

Copyright: American Physical Society http://www.aps.org/

Postprint available at: Linköping University Electronic Press http://urn.kb.se/resolve?urn=urn:nbn:se:liu:diva-114978 


\title{
Ultrafast dynamic conductivity and scattering rate saturation of photoexcited charge carriers in silicon investigated with a midinfrared continuum probe
}

\author{
Fanqi Meng, ${ }^{1}$ Mark D. Thomson, ${ }^{1}$ Bo E. Sernelius, ${ }^{2}$ Michael Jörger, ${ }^{3}$ and Hartmut G. Roskos ${ }^{1, *}$ \\ ${ }^{1}$ Physikalisches Institut, J. W. Goethe-Universität, Max-von-Laue-Strasse 1, 60438 Frankfurt am Main, Germany \\ ${ }^{2}$ Division of Theory and Modeling, Department of Physics, Chemistry and Biology, Linköping University, 58183 Linköping, Sweden \\ ${ }^{3}$ Bruker Optik GmbH, Rudolf-Plank-Strasse 27, 76275 Ettlingen, Germany
}

(Received 25 November 2014; published 4 February 2015)

\begin{abstract}
We employ ultrabroadband terahertz-midinfrared probe pulses to characterize the optical response of photoinduced charge-carrier plasmas in high-resistivity silicon in a reflection geometry, over a wide range of excitation densities $\left(10^{15}-10^{19} \mathrm{~cm}^{-3}\right)$ at room temperature. In contrast to conventional terahertz spectroscopy studies, this enables one to directly cover the frequency range encompassing the resultant plasma frequencies. The intensity reflection spectra of the thermalized plasma, measured using sum-frequency (up-conversion) detection of the probe pulses, can be modeled well by a standard Drude model with a density-dependent momentum scattering time of $\sim 200 \mathrm{fs}$ at low densities, reaching $\sim 20 \mathrm{fs}$ for densities of $\sim 10^{19} \mathrm{~cm}^{-3}$, where the increase of the scattering rate saturates. This behavior can be reproduced well with theoretical results based on the generalized Drude approach for the electron-hole scattering rate, where the saturation occurs due to phase-space restrictions as the plasma becomes degenerate. We also study the initial subpicosecond temporal development of the Drude response and discuss the observed rise in the scattering time in terms of initial charge-carrier relaxation, as well as the optical response of the photoexcited sample as predicted by finite-difference time-domain simulations.
\end{abstract}

DOI: 10.1103/PhysRevB.91.075201

PACS number(s): 78.47.-p

\section{INTRODUCTION}

The dynamics of charge carriers in silicon has been studied intensely over previous decades due to its importance in (opto-)electronic applications. Spectroscopy provides an important tool for fundamental investigations, as it allows one to elucidate the intraband charge-carrier dynamics via the frequency-dependent conductivity spectrum. For carriers in doped $\mathrm{Si}$, this response has been characterized over a very broad range of $n$-/ $p$-type dopant carrier concentration $\left(10^{15}-10^{20} \mathrm{~cm}^{-3}\right)$, employing measurements spanning the terahertz (THz) [1] and infrared [2] frequency ranges, where a Drude-type spectral behavior is generally observed, with modifications due to, e.g., inter-valence-band transitions and energy-dependent carrier relaxation rates. A number of reports have also been devoted to the study of photoexcited electronhole $(e-\mathrm{h})$ plasmas, not only because of their relevance for applications (optoelectronics [3], laser micromachining [4], and extreme surface nonlinear optics [5]) but also because they provide the possibility to study ultrafast relaxation/scattering processes vs excitation density $N_{\text {ex }}$ and energy $h v_{\text {ex }}$. Such pump-probe studies typically use interband excitation (UVnear-IR) and probe the spectral response in the $\mathrm{THz}[6,7]$, midinfrared (MIR) [8], or optical [9-12] range, depending on $N_{\text {ex }}$ (and hence the Drude plasma frequency $v_{\mathrm{p}}$ ). Other studies have estimated the time scales directly from time-domain data using an ultrashort optical probe pulse [13] or photoelectron detection [14]. Among these reports, various approaches are used to estimate the scattering time $\tau_{\mathrm{s}}=\Gamma^{-1}$ from the data. In reviewing the range of reported data (presented in this paper), we found that there is some disparity in the values and their dependence on $N_{\mathrm{ex}}$, and at high excitation densities

*Corresponding author: roskos@physik.uni-frankfurt.de
$\left(N_{\mathrm{ex}} \gtrsim 10^{20} \mathrm{~cm}^{-3}\right)$ there still appears to be contention about the order of magnitude for $\tau_{\mathrm{s}}$. For certain experiments (e.g., those involving the charge-carrier plasma as a moving front for Doppler up-conversion $[15,16])$, the precise magnitude of $\tau_{\mathrm{s}}$ sensitively affects the degree of absorption loss for probe frequencies (especially about $v_{\mathrm{p}}$ ), and hence a quantitative determination $\tau_{\mathrm{s}}$ vs $N_{\mathrm{ex}}$ is necessary. While the role of the various scattering mechanisms has been investigated theoretically [17-21], a detailed comparison between theory and experiment is also lacking.

In the present paper, we apply pump-probe spectroscopy on $\mathrm{Si}(100)$ at $T=293 \mathrm{~K}$ in a reflection geometry, using both ultrabroadband MIR-probe and conventional THz-probe pulses, to extract estimates of $\tau_{\mathrm{s}}$ (following initial energy relaxation) over the range $N_{\mathrm{ex}}=3 \times 10^{15}$ to $2 \times 10^{19} \mathrm{~cm}^{-3}$ by applying a standard Drude model to fit the intensity spectra (and accounting for the longitudinal excitation density profile in the analysis). A saturation in the increase of the scattering rate is observed for $N_{\mathrm{ex}} \sim 10^{19} \mathrm{~cm}^{-3}$, with $\tau_{\mathrm{s}} \sim$ $20 \mathrm{fs}$. This behavior can be reproduced well by theory, which includes the $e$-h scattering rate (whose variation dominates the density dependence in this range) via a generalized Drude approach $[19,21]$. This supports the assertion that the saturation is due to the onset of phase-space restrictions as the plasma becomes degenerate (and the density of vacant final states reduces). While the present time resolution is limited to a few hundred femtoseconds, we also investigate the subpicosecond evolution of the plasma response. A comparison to model results from one-dimensional (1D) finite-difference time domain (FDTD) simulations shows that while one should be wary of artifacts in the fitted values of $\tau_{\mathrm{s}}$ during the initial rise of the signal, the data indeed indicate that the scattering rate relaxes to a steady-state value during the first $1 \mathrm{ps}$ following excitation. 


\section{EXPERIMENTAL DETAILS}

The experimental system is based on a $1-\mathrm{kHz} \mathrm{Ti}: \mathrm{Al}_{2} \mathrm{O}_{3}$ amplifier laser (Clark-MXR, CPA-2101, $\lambda_{0}=775 \mathrm{~nm}$, $T_{\text {fwhm }}=150 \mathrm{fs}$ ). For the ultrabroadband THz-MIR probe pulses, we employ the emission from a two-color air plasma (as reported previously [22,23]), whose pump beam (energy $380 \mu \mathrm{J})$ is obtained by spectral broadening of the Chirped Pulse Amplification (CPA) laser pulses in an Ar-filled (2.5 bars) hollow-core fiber and subsequent recompression with a set of negative-dispersion mirrors. The pump beam was focused to generate the plasma with a plano-convex lens ( $f=$ $200 \mathrm{~mm}$ ) via a $150-\mu \mathrm{m}$ - thick beta barium borate (BBO) crystal $\left(32^{\circ}\right.$ cut $)$ to provide the second-harmonic beam. The ultrabroadband emission had a typical pulse energy of $100 \mathrm{~nJ}$ (directly after the plasma, as inferred from measurements with a calibrated pyroelectric detector) and spectral coverage extending to $\sim 150 \mathrm{THz}\left(5000 \mathrm{~cm}^{-1}, 0.62 \mathrm{eV}\right)$. This beam is collimated by an off-axis paraboloidal mirror (OAPM, effective focal length $f_{\text {eff }}=152.4 \mathrm{~mm}$ ) after passing a 450 $\mu \mathrm{m}-$ thick Si wafer (to discard the optical pump beams). The optical-pump THz-MIR-probe reflection measurements were performed by deflecting the probe beam with a 450$\mu \mathrm{m}-$ thick Si beam splitter to the sample, where it was focused by an OAPM ( $f_{\text {eff }}=101.6 \mathrm{~mm}$ ) at normal incidence, reflected, and transmitted through the Si beam splitter to the detection stage. The CPA sample-pump beam (with pulse energy up to $\sim 50 \mu \mathrm{J}$ ) was brought to a diameter of $0.6 \mathrm{~mm}$ (FWHM) on the sample, using a noncollinear geometry. For detection of the THz-MIR probe pulses, we employed sum-frequency (SF) generation (up-conversion) [24] in a $500-\mu \mathrm{m}-$ thick $\langle 110\rangle$-cut ZnTe crystal with 150-fs optical detection pulses, whereby the THz-MIR beam is collinearly focused with an OAPM $\left(f_{\text {eff }}=101.6 \mathrm{~mm}\right)$. Following the SF crystal, a notch filter is used to discard the input detection pulse, and the SF spectrum is measured by coupling free space into a miniature spectrometer with a cooled CCD sensor (Ocean Optics QE65). Integration times of 50-100 ms are sufficient to acquire a SF spectrum for each value of detection delay $t$. A measured reference spectrogram $S(v, t)$ of these pulses (where $v$ is the frequency offset from $v_{0}=c / \lambda_{0}$ ) is shown in Fig. 1(a). Here the beam was sent directly to the detection (and hence represents that which is incident on the sample for the measurements below). In order to estimate the intensity spectrum $I(v)$, we also calculate the frequency marginal $M(v)=\int \mathrm{d} t S(v, t)$, as shown in Fig. 1(b). Note that the spectrogram [and hence $M(v)$ ] is affected both by the phase-matching response [which for such thick crystals varies as $P\left(\nu, v_{0}\right) \sim 1 / \Delta k\left(\nu, v_{0}\right)$ [24]], as well as a convolution with the spectral optical detection spectrum (with width $\Delta v=$ $3.8 \mathrm{THz}$ here). Nevertheless, for the calculation of reflectivity spectra, $M(v)$ can be used with reasonable accuracy as long as the spectral resolution is included in subsequent analysis. One can see that the measured signal extends from the cutoff of the notch filter $(\sim 10 \mathrm{THz})$ up to $\sim 150 \mathrm{THz}$. The modulation in the spectrum is due to the absorption lines from ambient $\mathrm{H}_{2} \mathrm{O}$ ( $\sim 50$ and $110 \mathrm{THz})$ and $\mathrm{CO}_{2}(\sim 70 \mathrm{THz})$ in the beam path. While the temporal width of the spectrogram is dictated by the optical detection pulses, one can still extract an estimate of the group delay $T_{\mathrm{g}}(v)$ via the temporal first moment vs frequency,

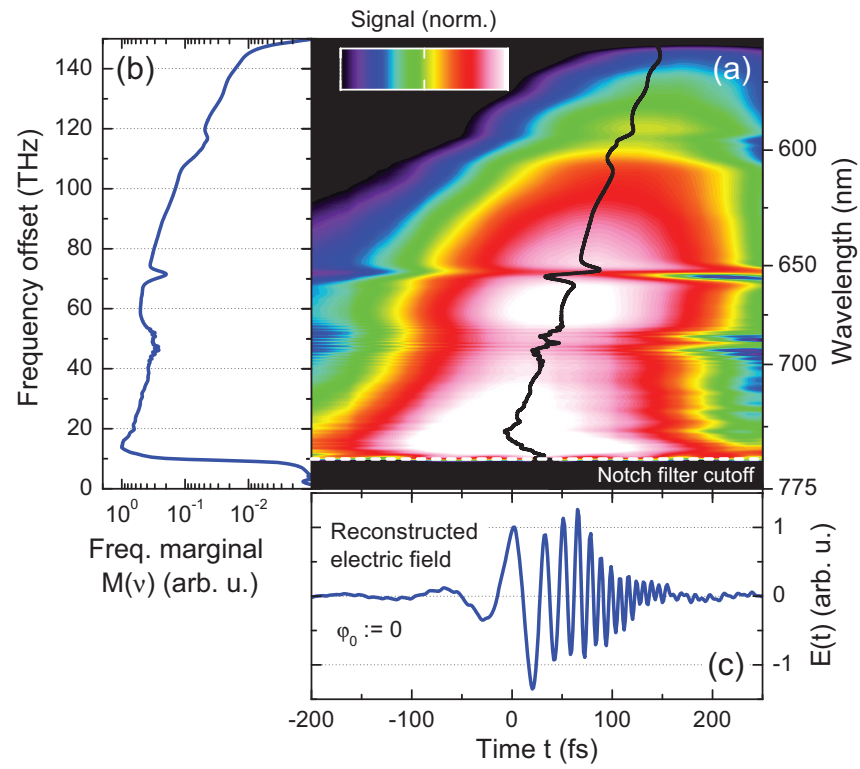

FIG. 1. (Color online) (a) Spectrogram of ultrabroadband THzMIR continuum probe pulse [black curve indicates temporal firstmoment vs frequency, $\bar{t}(v)]$. (b) Corresponding spectral marginal $M(v)$. (c) Estimate of temporal electric field profile $E(t)$, obtained using $M(v)$ and $\bar{t}(v)$ (assuming a carrier-envelope phase of $\varphi_{0}=0$; see text).

i.e., $\bar{t}(v)=\int d t t S(v, t) / \int d t S(v, t)$, which is also included in Fig. 1(a) (black line) and indicates a pulse duration of $\sim 100 \mathrm{fs}$, which is due primarily to the dispersion of the $450-\mu \mathrm{m} \mathrm{Si}$ wafer. While only approximate, to give an impression of the time-domain pulse, in Fig. 1(c) we plot a reconstructed field profile using the approximation $E(v) \sim \sqrt{M(\nu)} e^{-i \int_{0}^{v} d v \bar{t}(v)}$ (note that Sum Frequency Generation (SFG) detection can only provide the relative spectral phase, and we assume an arbitrary value of $\varphi_{0}=0$ for the carrier-envelope phase here to illustrate the temporal chirp of the carrier wave).

The purity of the high-resistivity $\mathrm{Si}$ samples used [float zone, (100), thickness of $525 \mu \mathrm{m}, \rho>3000 \Omega \mathrm{cm}$, Crystec $\mathrm{GmbH})$ was characterized by two different spectroscopic methods. First, shallow group-III and -V impurities were analyzed by low-temperature near-IR photoluminescence at $T=4.2 \mathrm{~K}$ using a Bruker Vertex80 FTIR spectrometer with a photoluminescence module and liquid-helium immersion cryostat. Quantification of impurities was carried out according to the SEMI MF1389 standard [25] using a suitable set of calibration samples. Excitation with two different wavelengths $\left(\lambda_{1}=532 \mathrm{~nm}\right.$, absorption depth $\alpha_{\mathrm{ex}} \sim 1 \mu \mathrm{m}$; $\left.\lambda_{2}=976 \mathrm{~nm}, \alpha_{\mathrm{ex}} \sim 70 \mu \mathrm{m}\right)$ gave similar results, indicating no significant gradient of the shallow impurity concentration. Second, substitutional carbon and interstitial oxygen content were analyzed according to the SEMI MF1391 and SEMI MF1188 standards with room-temperature MIR absorption spectroscopy using a Bruker Vertex70 FTIR spectrometer. The concentrations (upper bounds) of III/V dopants and C/O are listed in Table I, which indicates that the sample purity is not corrupted, e.g., by significant compensation doping compared to the excitation densities used here. 
TABLE I. Determined elemental impurity concentrations (in $\mathrm{cm}^{-3}$ ) in $\mathrm{Si}$ samples from low-temperature photoluminescence (4 K) and MIR absorption (300 K) measurements.

\begin{tabular}{lc}
\hline \hline Element & Concentration \\
\hline $\mathrm{B}$ & $\sim 2.9 \times 10^{11}$ \\
$\mathrm{P}$ & $\sim 7.9 \times 10^{11}$ \\
$\mathrm{C}$ & $<5 \times 10^{16}$ \\
$\mathrm{Al}$ & $<5 \times 10^{10}$ \\
$\mathrm{As}$ & $<5 \times 10^{10}$ \\
$\mathrm{O}$ & $<5 \times 10^{16}$ \\
$\mathrm{Sb}$ & $<5 \times 10^{10}$ \\
\hline
\end{tabular}

\section{RESULTS}

\section{A. Excitation density dependence}

Figure 2(a) shows the measured intensity reflection spectra $R(\nu, \tau) / R_{0}(v)$ (i.e., relative to the reflection $R_{0}$ of the unpumped sample, which is a flat curve with $R_{0}=0.30$ ) at a pump-probe delay of $\tau=1 \mathrm{ps}$ and a range of front-face $(z=0)$ excitation densities $N_{\mathrm{ex}}=0.35-2.1 \times 10^{19} \mathrm{~cm}^{-3}$.

Each reflection spectrum was fitted assuming a standard Drude model for the plasma conductivity $\sigma(\omega, z)=$ $e^{2} N_{\text {ex }}(z) / m_{\text {eff }}\left(1+i \omega \tau_{\mathrm{s}}\right)^{-1}$, where $m_{\text {eff }}=0.15 m_{0}$ is taken for the effective reduced $e$-h mass and $N_{\mathrm{ex}}(z)=N_{\mathrm{ex}} e^{-\alpha_{\mathrm{ex}} z}$ accounts for the $z$-dependent longitudinal excitation. It is well established for the reflectivity of such an excitation profile (and probe wavelengths $\lambda=c / \nu \gtrsim \alpha_{\mathrm{ex}}^{-1}$ ) that one should not use simply the Fresnel field reflection coefficient $r_{\mathrm{F}}$ (i.e., based on the front-face density) for quantitative analysis; rather, one must account for the distributed reflection of (and losses within) the excitation profile [26,27]. The solution for the total field reflectivity at normal incidence can still be expressed in closed form, which upon inspection of the formulas in Ref. [26], can be expressed as $r=$ $\left(1-n^{\prime}\right) /\left(1+n^{\prime}\right)$, where $n^{\prime}=n_{\mathrm{b}}+\sqrt{\Delta \epsilon_{\mathrm{r} 0}} X_{\beta}(\xi), n_{\mathrm{b}}=\sqrt{\epsilon_{\mathrm{br}}}$ is the background (unpumped) refractive index, $\Delta \epsilon_{\mathrm{r} 0}(\omega)=$ $-i \sigma(\omega, 0) / \epsilon_{0} \omega$ is the front-face photoinduced change in the complex permittivity, and $X_{\beta}(\xi)=I_{\beta+1}(\xi) / I_{\beta}(\xi)$ is the ratio of modified Bessel functions with $\beta=2 i \omega n_{\mathrm{b}}\left(c \alpha_{\mathrm{ex}}\right)^{-1}$ and $\xi=2 i \omega \sqrt{\Delta \epsilon_{\mathrm{r} 0}}\left(c \alpha_{\mathrm{ex}}\right)^{-1}$. During the fitting, we convolved the raw model spectra with the spectral detection response (width $\Delta v$ given above).

The fitted model curves are included in Fig. 2(a) and are generally in close agreement with the experimental data. The corresponding fit parameters $\left[\tau_{\mathrm{s}}\right.$ and $\nu_{\mathrm{p}}=\omega_{\mathrm{p}} / 2 \pi$, where $\left.\omega_{\mathrm{p}}^{2}=\sigma(0,0) / \epsilon_{\mathrm{br}} \epsilon_{0}\right]$ are shown in Fig. 2(b). We first note that the values of $v_{\mathrm{p}}$ correspond closely to a $\sqrt{N_{\mathrm{ex}}}$ dependence (solid curve), which supports the idea that $N_{\mathrm{ex}} \propto F$ (where $F$ is the excitation fluence) and shows that higher-order excitation effects (e.g., two-photon absorption [12]) do not play a role in these measurements. The values of the Drude scattering time $\tau_{\mathrm{s}}$ exhibit a moderate trend of increasing scattering rate $\Gamma=\tau_{\mathrm{s}}^{-1}$ with $N_{\mathrm{ex}}$, ranging from $\tau_{\mathrm{s}}=30.7 \mathrm{fs}\left(N_{\mathrm{ex}}=\right.$ $\left.0.35 \times 10^{19} \mathrm{~cm}^{-3}\right)$ to $\tau_{\mathrm{s}}=19.7 \mathrm{fs}\left(N_{\mathrm{ex}}=2.1 \times 10^{19} \mathrm{~cm}^{-3}\right)$, as discussed below. We note that the fit residuals in Fig. 2(a) typically deviated beyond the random noise levels in the data; that is, a small systematic disagreement exists for each data
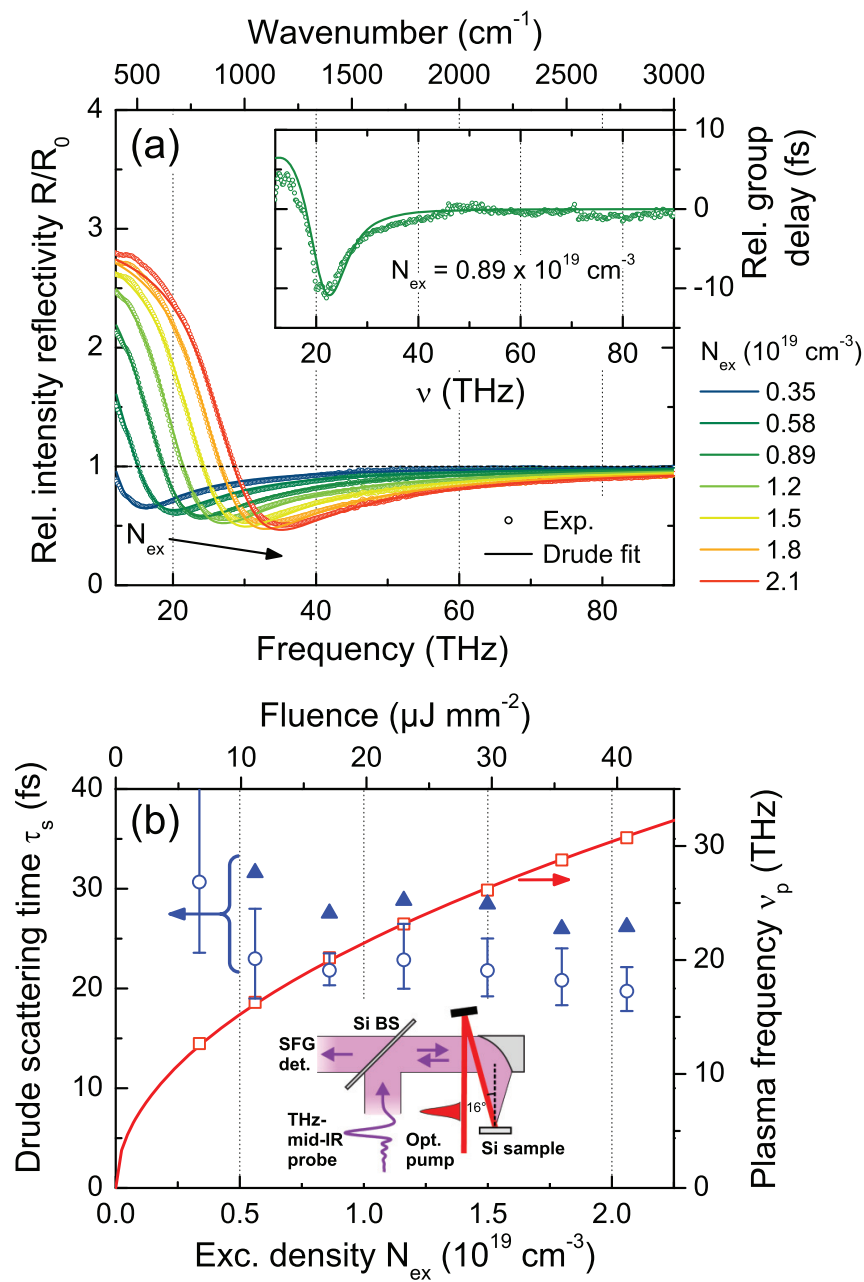

FIG. 2. (Color online) (a) Experimental intensity reflection spectra $R(v)=M(v) / M_{0}(v)$ for different values of optically excited plasma density $N_{\mathrm{ex}}\left(\lambda_{\mathrm{ex}}=775 \mathrm{~nm}\right)$ and a pump-probe delay of $\tau=1 \mathrm{ps}$ and fitted model curves assuming a standard Drude dispersion model and accounting for the finite-depth exponential excitation density profile (with $\alpha_{\mathrm{ex}}=125 \mathrm{~cm}^{-1}$ ). The inset shows the corresponding group delay $\Delta \bar{t}(v)$ and model curve using parameters from fitting intensity reflection. (b) Corresponding parameters $\tau_{\mathrm{s}}$ (circles) and $v_{\mathrm{p}}$ (squares) from model fits in (a) vs $N_{\mathrm{ex}}$. Also included are fitted values of $\tau_{\mathrm{s}}$ (triangles) when using only the SF spectra at $t=0$, for later discussion in connection with the data in Fig. 4. The inset depicts a schematic of experimental reflection geometry.

set, precluding a standard fit-parameter error analysis [28]. In order to obtain confidence intervals for the fitted values of $\tau_{\mathrm{s}}$, we analyzed the rms misfit $O=\sqrt{\sum\left(R-R_{\text {mod }}\right)^{2}}$ in the $\left(v_{\mathrm{p}}, \tau_{\mathrm{s}}\right)$ neighborhood of the fitted parameters and give here the bounds for $\tau_{\mathrm{s}}$ for the elliptical region where $O=\sqrt{2} O_{\min }$. Note that while we fitted here only the intensity curves $R(v)=|r(v)|^{2}$, for the field reflectivity response $r(v)$, one also expects a spectral phase corresponding to a small but measurable group delay $\Delta T_{\mathrm{g}}(\nu)$. This can also be estimated from the spectrograms via $\Delta \bar{t}(v)=\bar{t}(v)-\bar{t}_{0}(v)$, as described above. In the inset of Fig. 2(a) we plot the experimental $\Delta \bar{t}(v)$ data and the model curve obtained using the intensity-fit parameters for the case $N_{\mathrm{ex}}=0.89 \times 10^{19} \mathrm{~cm}^{-3}$, which are seen to be in good agreement and further demonstrate that 


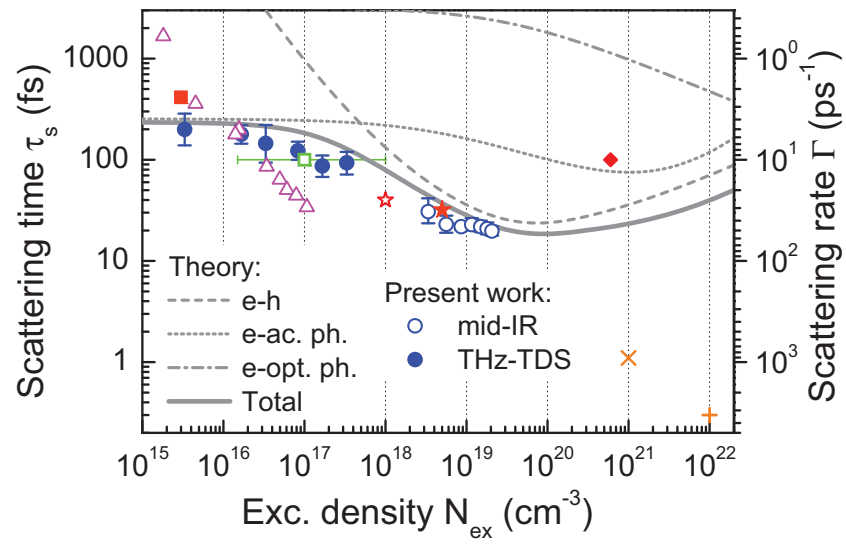

FIG. 3. (Color online) Experimental and theoretical (Drude) scattering time vs $N_{\text {ex }}$. Values from present work $\left(\lambda_{\text {ex }}=775 \mathrm{~nm}\right)$ using THz-TDS (solid circles; see text) and MIR continuum probe with SFG detection [open circles; data from Fig. 2(b)]. Literature sources (including $\lambda_{\mathrm{ex}}$, at room temperature unless otherwise stated): Ref. [6] (solid square, $760 \mathrm{~nm}$ ), Ref. [7] (triangles, $400 \mathrm{~nm}, T=$ 30 K), Ref. [29] (open square, 800 nm), Ref. [14] (open star, 610 nm), Ref. [13] (solid star, $800 \mathrm{~nm}$ ), Ref. [30] (diamond, $530 \mathrm{~nm}$ ), Ref. [12] (cross, $625 \mathrm{~nm}$ ), and Ref. [10] (plus, $620 \mathrm{~nm}$ ). Curves correspond to theoretical calculations of the total scattering rate (solid) from $e$-h (dashed) [21], acoustic-phonon (dotted), and optical-phonon (dash-dot) scattering [17] (see text).

the experimental data correspond well to the Drude/reflection model used.

In order to put these measured values of $\tau_{\mathrm{s}}$ in perspective, in Fig. 3 we plot the values along with various previously reported estimates from the literature (as indicated in the caption) vs $N_{\mathrm{ex}}$. In order to provide additional data for lower $N_{\text {ex }}$, we also performed a set of optical-pump-THzprobe reflection measurements using a conventional $\mathrm{THz}$ time-domain spectroscopy (TDS) system covering the range 0.1-3 THz. The obtained values of $\tau_{\mathrm{s}}$ (for $N_{\mathrm{ex}}=3.3 \times 10^{15}$ to $3.3 \times 10^{17} \mathrm{~cm}^{-3}$ at $\tau=+5 \mathrm{ps}$ ) are also included in Fig. 3 [where we applied the same model as above, except here fitting the complex reflectivity data $r(v)]$. The literature data were obtained using various techniques, some of which do not directly probe precisely the Drude scattering, i.e., current damping, rate, including reports in which the values are extracted directly from analysis and those in which estimates are cited that are found to be consistent, e.g., with the results of auxiliary simulations (and as such may indicate only an approximate value of $\tau_{\mathrm{s}}$ ).

For low excitation densities, our results give a value of $\tau_{\mathrm{s}}=200 \pm 75 \mathrm{fs}$, which is close to cited values for the $e$-ph scattering time $\Gamma_{\mathrm{e}-\mathrm{ph}}^{-1}$ at $T=300 \mathrm{~K}$ of $240-260 \mathrm{fs}$ [13,14,31]. A previous pump-probe THz-TDS study (solid square in Fig. 3, $N_{\mathrm{ex}}=3 \times 10^{15} \mathrm{~cm}^{-3}[6]$ ) estimated a value of $\tau_{\mathrm{s}}=330-500 \mathrm{fs}$ $(\Gamma / 2 \pi=2-3 \mathrm{THz})$, although that report concentrated on simulating the response during the initial dynamics (discussed further below) and accounting for the finite pulse duration of the $\mathrm{THz}$ probe pulse, as opposed to a rigorous analysis of the conductivity spectra of a quasistatic plasma at sufficiently large delay $\tau$. A more recent study using THz-TDS [7] at $T=30 \mathrm{~K}$ with $\lambda_{\mathrm{ex}}=400 \mathrm{~nm}$ (triangles) fitted the complex conductivity spectra $\sigma(v)$ for $\tau=+10 \mathrm{ps}$ as a function of $N_{\text {ex }}$. Given the low temperature, the higher $\tau_{\mathrm{s}}$ values $(\sim 2 \mathrm{ps})$ at low densities are reasonable, as $e$-ph scattering is strongly suppressed [32]. However, the observed trend with $N_{\text {ex }}$ differs significantly from our results, as discussed further below. Our data are consistent with the value of $\tau_{\mathrm{s}}=100 \mathrm{fs}$ employed in Ref. [29] (open square) to simulate experiments involving the reflection from a counterpropagating plasma boundary in the range $N_{\mathrm{ex}}=1.5 \times 10^{16}-10^{18} \mathrm{~cm}^{-3}$ (although no error margin for this value was given)

In the range $N_{\mathrm{ex}}=10^{18}-10^{19} \mathrm{~cm}^{-3}$, two reports provide estimates of carrier scattering time which are reasonably consistent with our data, although they do not specifically probe the current relaxation rate. In Ref. [14] (open star, $N_{\mathrm{ex}} \sim$ $10^{18} \mathrm{~cm}^{-3}$ ), optical two-photon photoemission measurements were used to deduce an initial time scale for momentum relaxation of $\tau_{\mathrm{s}}=40 \mathrm{fs}$, whereas in Ref. [13] (solid star, $N_{\mathrm{ex}}=$ $5.5 \times 10^{18} \mathrm{~cm}^{-3}$ ) a time scale of $\tau_{\mathrm{s}}=32 \pm 5 \mathrm{fs}$ was determined from the decay of the coherent four-wave-mixing signal. In both of these reports, this time scale accounts specifically for the initial (elastic) momentum reorientation and redistribution of the carriers, while the energy relaxation time (due to $e$-ph scattering) was determined through additional measurements to be $\tau_{\mathrm{e}-\mathrm{ph}} \sim 250 \mathrm{fs}$. Hence these studies include the $e$-h and $e-e$ scattering contributions directly, the latter of which is expected to contribute only to the Drude current damping via the conduction-band anisotropy [21]. We note that another study [8] of MIR reflectivity spectra (similar to the MIR measurements here, except with a pointwise probing with tunable source) for $N_{\mathrm{ex}} \sim 10^{19}-10^{20} \mathrm{~cm}^{-3}$ used a nominal value of $\tau_{\mathrm{s}}=100 \mathrm{fs}$ to provide a set of additional simulated curves. However, as no attempt was made to extract $\tau_{\mathrm{s}}$ from the experimental data, we omit this value in Fig. 3.

The remaining data at higher excitation density $N_{\mathrm{ex}}>$ $10^{20} \mathrm{~cm}^{-3}$ all involved using a single probe wavelength and varying $N_{\mathrm{ex}}$ for determining the Drude response. The value of $\tau_{\mathrm{s}}=100$ fs in Ref. [30] (diamond, $N_{\mathrm{ex}} \sim 6 \times 10^{20} \mathrm{~cm}^{-3}$ ), which tends to deviate significantly from the overall trend of the data, was cited in connection with detailed simulations of the delay-dependent reflectivity of a 20-ps probe pulse $(\lambda=2.8 \mu \mathrm{m}$ ), although no estimate of the error was provided and perhaps should be taken to indicate only the order of magnitude of $\tau_{\mathrm{s}}$. The extreme excitation densities (and hence $v_{\mathrm{p}}$ values) of Ref. [12] (cross, $N_{\mathrm{ex}} \sim 10^{21} \mathrm{~cm}^{-3}$ ) and Ref. [10] (plus, $N_{\mathrm{ex}} \sim 10^{22} \mathrm{~cm}^{-3}$ ) required the use of a visible-range probe, with estimates $\tau_{\mathrm{s}} \sim 1.1$ and 0.3 fs obtained by fitting the Drude-type reflection curves, respectively. While the fitted curves around $v=v_{\mathrm{p}}$ in those reports are in reasonable agreement with the experimental data (although in both reports there are deviations on the high- $N_{\text {ex }}$ side), it is not clear whether such an approach can be applied robustly to extract $\tau_{\mathrm{s}}$. In simulations, we found that the extracted value of $\tau_{\mathrm{s}}$ from reflectivity spectra is particularly sensitive to any distortion of the curves, as it is essentially the curvature of $R(v)$ about $v_{\mathrm{p}}$ which allows its determination. Given that a Drude analysis of data with a single probe wavelength necessitates the variation of $N_{\mathrm{ex}}$ (and hence $\tau_{\mathrm{s}}$ ), distortions of the measured $R\left(N_{\mathrm{ex}}\right)$ curves are to be expected [compared to spectra $R(v)$ at constant $N_{\text {ex }}$ ], and we exercise caution in considering these values. We note that in both reports, it is the 
initial subpicosecond carrier plasma response that is probed; that is, in Ref. [12] $R\left(N_{\mathrm{ex}}\right)$ is measured with a pump-probe delay of $\tau=150 \mathrm{fs}$ with 100-fs pulses, while in Ref. [10] the self-induced reflection changes are measured with 90 -fs pulses. However, in a preceding experimental report [11], the authors of Ref. [12] and colleagues extracted $\tau_{\mathrm{s}}$ from their data vs pump-probe delay (for a fixed $N_{\mathrm{ex}} \sim 10^{22} \mathrm{~cm}^{-3}$ ), asserting that $\tau_{\mathrm{s}} \approx 0.5$ fs for the whole measured delay range out to $\tau=0.8 \mathrm{ps}$. Note that for those measurements, a single probe wavelength was used with an analysis of $p$ and $s$ reflectivity at oblique incidence to extract the complex refractive index and hence Drude parameters, as opposed to analysis of $R\left(N_{\mathrm{ex}}\right)$ curves. To summarize, while some concern has been raised about the plausibility of these values [21], the assertion in the literature that scattering times $\tau_{\mathrm{s}} \sim 1 \mathrm{fs}$ are obtained at these high densities (even for time scales $\gtrsim 100 \mathrm{fs}$ ) has not yet been refuted.

\section{B. Theoretical predictions}

We now turn to theoretical predictions for the observed dependence on $N_{\mathrm{ex}}$. Qualitatively, the $e$-phonon scattering rate $\Gamma_{\mathrm{e}-\mathrm{ph}}$ is expected to vary only weakly with $N_{\mathrm{ex}}$ for densities below $\sim 10^{20} \mathrm{~cm}^{-3}$ [32], whereas (bimolecular) $e-\mathrm{h}$ scattering should obey $\Gamma_{\mathrm{e}-\mathrm{h}} \propto N_{\mathrm{ex}}$ for low densities. While like-charge scattering does not contribute to current damping for isotropic bands, for the anisotropic $\mathrm{X}$ valleys in $\mathrm{Si}, e-e$ scattering can also contribute, although in the low-frequency limit this is predicted to have only a very minor influence on the measured scattering time [21]. The situation for increasing $N_{\text {ex }}$ was closely examined theoretically [17,19,21] following the subfemtosecond experimental value found in Ref. [10]. Using different approaches, they all predict that as the plasma becomes degenerate $\left[N_{\mathrm{ex}} \gtrsim N_{\mathrm{ex}}^{\mathrm{thr}}\right.$, where $N_{\mathrm{ex}}^{\mathrm{thr}}=$ $\left(2 m_{\mathrm{de}} k T / \hbar^{2}\right)^{3 / 2} / 3 \pi^{2}$, [17]] $\Gamma_{\mathrm{e}-\mathrm{h}}$ should reach a maximum value and then begin to decrease with $N_{\text {ex }}$ due to the reduction of available final states for scattering (phase-space restrictions in the quantum limit [19]). For electrons in the conduction band at $T=300 \mathrm{~K}$ one has $N_{\mathrm{ex}}^{\mathrm{thr}}=3.4 \times 10^{18} \mathrm{~cm}^{-3}$, using the value $m_{\mathrm{de}}=0.32 m_{\mathrm{e}}$ for the (valley-degeneracy-scaled) density of states mass. Although the effective temperature $T$ can be expected to rise with excitation fluence due to the excess excitation photon energy (for $h v_{\mathrm{ex}}>E_{\mathrm{g}}=1.12 \mathrm{eV}$ ), at $T=300 \mathrm{~K}$ this amounts to only some $10 \mathrm{~K}$ for $h \nu_{\mathrm{ex}}=1.6 \mathrm{eV}$. Hence one expects that the $e$-h scattering will dominate the density dependence of $N_{\mathrm{ex}}$ and that a saturation of the $\Gamma \propto N_{\mathrm{ex}}$ dependence should be observed for $N_{\mathrm{ex}} \sim 10^{18}-10^{19} \mathrm{~cm}^{-3}$, as is indeed indicated in our experimental results.

We carried out calculations of $\Gamma_{\mathrm{e}-\mathrm{h}}$ vs $N_{\mathrm{ex}}$ using the theoretical treatment in Ref. [21], i.e., based on a generalized Drude model, where the $e$-h scattering is calculated from integration over the appropriate expression of the complex polarizability functions $\alpha_{\mathrm{e}, \mathrm{h}}(\mathbf{q}, \omega)$ to yield the relevant coefficients of mutual friction, which are then used to evaluate the complex conductivity $\sigma(v)$. Here we assume isotropic bands for simplicity (with effective masses $m_{\mathrm{de}}=0.32 m_{\mathrm{e}}$ and $\left.m_{\mathrm{h}}=0.52 m_{\mathrm{e}}\right)$ and calculate the low-frequency limit for $\Gamma_{\mathrm{e}-\mathrm{h}}$ (which is a reasonable approximation for the spectral range used in our experiments) using the finite-temperature $(T=300 \mathrm{~K})$ expression for $\operatorname{Im}\left\{\alpha_{\mathrm{e}, \mathrm{h}}\right\}$ and the zero-temperature expression for $\operatorname{Re}\left\{\alpha_{\mathrm{e}, \mathrm{h}}\right\}$. The calculated rates $\Gamma_{\mathrm{e}-\mathrm{h}}$ vs $N_{\mathrm{ex}}$ are shown in Fig. 3 (dashed curve) and indeed exhibit a saturation of the $\Gamma_{\mathrm{e}-\mathrm{h}} \propto N_{\mathrm{ex}}$ dependence in the range mentioned above, reaching a minimum with $\tau_{\mathrm{s}} \sim 20$ fs at $N_{\mathrm{ex}}=8 \times 10^{19} \mathrm{~cm}^{-3}$, above which the $e$-h scattering rate begins to drop again.

In order to include the contribution of $e$-ph scattering, we employed the theoretical expressions in Ref. [17] [Eqs. (7) and (8) for the optical and acoustic phonon scattering, respectively]. The calculated curves are also shown in Fig. 3 (using the following values: LO optical phonon deformation potential $D_{1}=6 \times 10^{8} \mathrm{eV} \mathrm{cm}^{-1}$ [33], optical phonon energy $\hbar \Omega_{\mathrm{oph}}=64.4 \mathrm{meV}$ [34], acoustic deformation potential $E_{1}=$ $8.2 \mathrm{eV}$ [35], sound velocity $v_{\mathrm{s}}=9 \times 10^{5} \mathrm{~m} \mathrm{~s}^{-1}$ ). As expected, the acoustic phonon scattering rate $\Gamma_{\mathrm{e}-\mathrm{aph}}$ dominates in the low-density limit, with a value $\tau_{\mathrm{s}} \approx 250$ fs reproducing experimental values well. Based on this model, $\Gamma_{\mathrm{e}-\text { aph }}$ increases gradually (with a $N_{\mathrm{ex}}^{1 / 3}$ dependence due to an increase of the electron velocity), reaching a maximum at $N_{\mathrm{ex}} \approx 10^{21} \mathrm{~cm}^{-3}$, upon which it decays with $N_{\mathrm{ex}}^{-1}$ due to the additional onset of phase-space restrictions (as per $\Gamma_{\mathrm{e}-\mathrm{h}}$ above). The contribution of optical phonon scattering is marginal, increasing only at very high densities where electrons in the distribution increasingly reach energies $\sim \hbar \Omega_{\text {oph }}$.

The combined scattering rate is also shown in Fig. 3 (solid curve) and is seen to reproduce the trend and magnitude of our experimental data well. To our knowledge, such a correspondence between experiment and theory for the Drude scattering time for carrier plasmas in undoped $\mathrm{Si}$ vs $N_{\text {ex }}$ has not been demonstrated until now. This correspondence has an important impact for judging the overall trend in the experimental data in Fig. 3. On the basis of the experimental data alone, one might consider that the very low reported values of $\tau_{\mathrm{s}}$ for $N_{\mathrm{ex}} \geqslant 10^{21}$ could be consistent with a $\Gamma \propto N_{\mathrm{ex}}$ dependence extending to these high densities (as has been previously asserted [10]), with the apparent saturation of the values from our measurements for $N_{\mathrm{ex}} \sim 10^{19} \mathrm{~cm}^{-3}$ possibly being due to unforeseen artifacts in the experimental method. However, the consistency in the present results seems to rule this out, at least for charge-carrier plasmas after any carrier-carrier thermalization on a sub-100-fs time scale (see the next section). Clearly, the treatment here of $e$-h and $e$-ph scattering does not predict scattering times of $\tau_{\mathrm{s}} \sim 1$ fs at high density. We also have difficulties with reconciling the data from Ref. [7] (Fig. 3, triangles). In considering the low temperature for those measurements, one expects the onset of plasma degeneracy to be at densities even lower than those in our experiments. Further experiments vs $T$ and excitation energy are required to resolve this issue, in which a broader probe spectral range including the plasma frequency is covered, as per our measurements here.

\section{Subpicosecond relaxation dynamics}

In this last section, we present time-resolved measurements of the plasma response probed with the THz-MIR pulses. As will be shown, while certain aspects of the data would necessitate time resolution superior to that available here, we can draw important conclusions regarding the analysis 
and interpretation of such measurements via comparison with simulations.

The time-resolved THz-MIR reflectivity spectra $R^{\prime}(\nu, \tau)$ were measured in the same experimental geometry as the data in Fig. 2(a) with $N_{\mathrm{ex}}=2.1 \times 10^{19} \mathrm{~cm}^{-3}$, except here, in order to achieve practical measurement times, we acquired each SF spectrum $S\left(v, t_{0} ; \tau\right)$ at a fixed time $t=t_{0}$, as opposed to acquiring a full spectrogram to calculate the marginal (hence the measurements capture the spectral components within the 150-fs SF detection gate pulse). The data are shown in Fig. 4(a). For each spectrum, we employed the same Drude model analysis as above in order to yield $N_{\text {ex }}$ and $\tau_{\mathrm{s}}$ as a function of $\tau$. The model spectra and corresponding fit parameters are shown in Figs. 4(b) and 4(c), respectively. As can be seen, the data can be reproduced well over the full delay range with this model. The kinetics of the fitted $N_{\text {ex }}$ data can be fit well assuming a Gaussian response (i.e., error-function dependence) with a FWHM width of $T_{\mathrm{r}}=420 \mathrm{fs}$ (due to the combination of the pump-probe correlation function and noncollinear geometry). The corresponding kinetics of the $\tau_{\mathrm{s}}$ values show a rise from $\tau_{\mathrm{s}} \sim 17$ fs around $\tau=0$ to a value of $\tau_{\mathrm{s}}=28 \mathrm{fs}$ for a delay of some picoseconds. The continuing rise of the $\tau_{\mathrm{s}}$ curve compared to that of $N_{\mathrm{ex}}$ clearly indicates a subsequent evolution of the plasma scattering rate following excitation. This trend is not consistent with that expected simply for an increasing plasma density during the excitation pulse and is addressed in detail below. A comparison of the value for $\tau=+1 \mathrm{ps}\left(\tau_{\mathrm{s}} \approx 26 \mathrm{fs}\right.$ ) with the corresponding data in Fig. $2(\mathrm{~b})\left(\tau_{\mathrm{s}} \approx 19.7 \mathrm{fs}\right)$ reveals an apparent discrepancy (both sets of data were acquired in the same measurement run). As the only difference in the two measurements is that in one case the full SF marginal $M(v)$ is acquired vs the SF spectra at fixed $t=t_{0}$, as a first step to address this issue we reanalyzed the data in Fig. 2 by extracting only the single spectra $S\left(\nu, t_{0}\right)$ from the full spectrogram. The resultant fitted values of $\tau_{\mathrm{s}}$ are also included in Fig. 2(b) (solid triangles) and are indeed systematically larger than those obtained with the full spectrogram marginals [and thus are consistent with the value in Fig. 4(c) for $N_{\mathrm{ex}}=2.1 \times 10^{19} \mathrm{~cm}^{-3}$ ]. Hence an important finding is that SF time gating introduces artifacts in such measurements; we explain the reason for this below with the help of simulation results.

We modeled the experiments using 1D FDTD simulations, with a Drude response and spatiotemporal profile for the photoinduced plasma (as described previously [15,16], except here with copropagating pump-probe pulses and front-face excitation). Simulation batches were carried out vs the pumpprobe delay $\tau$, using a time-independent nominal scattering time of $\tau_{\mathrm{s} 0}=25 \mathrm{fs}$. The numerical reflected fields were then analyzed to produce either the full spectrum or that corresponding to SF detection at a fixed time $t_{0}$ (with a 150 -fs SF detection gate), corresponding to the various experimental results here. These spectra were then fitted using the same Drude model algorithm. We present the results for three sets of simulation conditions in order to isolate various aspects expected in the analysis of the experimental data, as shown in Fig. 4(d) [curves (i)-(iii)].

In case (i), we used a transform-limited (TL) $\mathrm{THz}-$ MIR probe pulse with an intensity spectrum based on the experimental one (Fig. 1) and a pump pulse duration equal
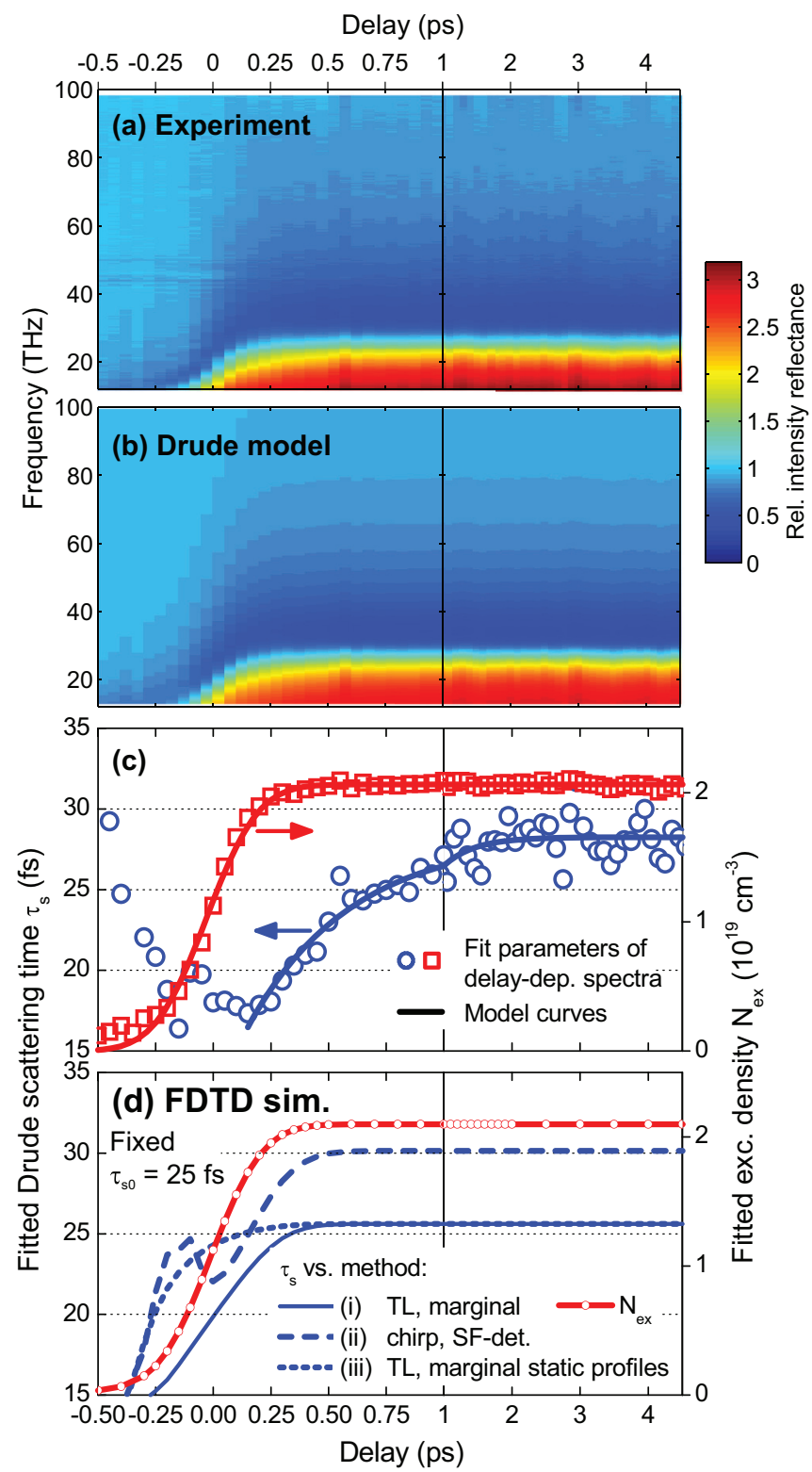

FIG. 4. (Color online) (a) Experimental relative intensity reflection spectra vs pump-probe delay $\tau$, estimated using SF spectra at fixed detection time $t_{0}$, i.e., $R^{\prime}(\nu, \tau)=S\left(v, t_{0} ; \tau\right) / S_{0}\left(\nu, t_{0}\right)$, where $S_{0}(v)$ is the reference spectrum before excitation (measured at sufficiently negative $\tau$ ). (b) Corresponding model spectra using Drude model fits for each value of $\tau$. (c) Fitted Drude parameters $\tau_{\mathrm{s}}$ (circles) and $N_{\text {ex }}$ (squares) for model data in (b). (d) Corresponding analysis of FDTD simulation results with a constant value of $\tau_{\mathrm{s} 0}=25 \mathrm{fs}$, demonstrating artifact rise of fitted $\tau_{\mathrm{s}}$ values around zero delay for three simulation batches: (i) transform-limited (TL) THz-MIR pulse with full spectra (solid line), (ii) including experimental THz-MIR pulse chirp and simulating SF detection at fixed delay (long-dashed line), and (iii) with a TL probe pulse and a sequence of static, partially formed plasma profiles (short-dashed line).

to the experimentally observed $T_{\mathrm{r}}$ for $N_{\mathrm{ex}}$ in Fig. 4(c) and fitted the full spectra of the numerical reflected THz-MIR pulses for each $\tau$. The kinetics of the fitted $N_{\mathrm{ex}}$ values are as expected and correspond closely to the experimental data. Around zero delay, the fitted values of $\tau_{\mathrm{s}}$ are also seen to 
rise from $\lesssim 15$ fs toward the simulation value of $\tau_{\mathrm{s} 0}=25$ fs (note the slight error is due to the finite accuracy of the FDTD simulations). However, it is also apparent that this artificial rise follows the same delay dependence as the $N_{\text {ex }}$ curve; that is, the effect occurs only during the formation of the plasma when there is temporal overlap between pump and probe pulses.

Case (ii) adds the additional effects of our measurements in Figs. 4(a)-4(c) by adding the experimental chirp $\left(\varphi^{\prime \prime}=126 \mathrm{fs}^{2}\right)$ to the input THz-MIR pulse and using the numerically time-gated SF spectra. This leads again to a rise in the fitted $\tau_{\mathrm{s}}$ curves, albeit with a more complex variation during the leading portion of the excitation pulse, $\tau \lesssim 0$. [Note the fitted $N_{\text {ex }}$ curve is essentially the same for all simulation cases, and only that for case (i) is shown.] However, in this case, one sees that fitted $\tau_{\mathrm{s}}$ values in the steady state are systematically larger than the underlying value of $\tau_{\mathrm{s} 0}$ used in the simulations (as seen in the experimental analysis above). This is not the case when the full spectra are used (with either TL or chirped THz-MIR pulses; the latter results are not shown). This allows us to identify the origin of the discrepancy above: the effect of the finite SF-detection gate pulse introduces a small distortion on the measured spectra which results in a systematic error (overestimation) in the fitted value of $\tau_{\mathrm{s}}$, despite that fact that the gate is also applied to the detection of the reference pulse (without plasma excitation). With the detailed simulation results at hand, this can be traced to the small group-delay variation experienced by the THz-MIR pulse in reflection [see inset in Fig. 2(a)]. While this is of the order of only $\pm 10 \mathrm{fs}$, it is indeed sufficient to distort the spectra in this way. This is an important result, as one may have expected that the use of a reflection geometry would remove any influence of plasma dispersion (compared to bulk delays in transmission), and emphasizes the role of the finite group-delay in reflection from (and associated penetration into) a Drude medium. In future investigations this effect could be alleviated by the use of a significantly longer SF detection pulse (i.e., $T_{\mathrm{SF}} \rightarrow 1 \mathrm{ps}$ via spectral filtering). Nevertheless, one sees that the rise of the fitted $\tau_{\mathrm{s}}$ curve for case (ii) still is essentially complete after the pump pulse excitation and that these effects alone do not account for the slower rise in the experimental data [Fig. 4(c)].

Now that we have demonstrated numerically that such experiments inherently produce artifacts in the fitted value of $\tau_{\mathrm{s}}$ around zero delay (which could cause physical misinterpretation of data, even with superior time resolution), the question of its specific cause remains. About zero delay there are two deviations from the steady-state situation assumed in the Drude model here (besides the possibility of a timedependent $\tau_{\mathrm{s}}$ value); that is, not only does the THz-MIR pulse copropagate with the pump pulse and experience a time-nonstationary medium, but also the excitation profile assumed in the model (exponential decay with $z$ ) is not yet fully formed, and hence the expression used for calculating $r(v)$ (see Sec. III A [26]) is not completely accurate. In order to isolate these two factors, we also performed a simulation batch [Fig. 4(d), case (iii)] with a control situation in which the plasma for each value of delay is kept artificially "frozen" at its profile for $t=\tau$ (and the THz-MIR pulse reflects from a time-stationary, partially formed plasma profile). As can be seen from the fitted $\tau_{\mathrm{s}}$ curve (iii), while one still observes an artifact rise (due to the inaccuracy of the plasma profile model), this is now significantly more abrupt. Hence one may conclude that the copropagation and time-nonstationary medium dominate the slower rise seen in the curve for case (i). This is an important result for the interpretation of future experiments, as this effect will be present even for much shorter pump and probe pulses, and one should also employ detailed simulations of the copropagation to avoid misinterpretation of experimental data.

Finally, returning to the delay dependence of $\tau_{\mathrm{s}}$ in Fig. 4(c), in Fig. 4(d) we provide a fit of the data (for $\tau \geqslant 200 \mathrm{fs}$ ) with an exponential step function $\propto\left(1-e^{-\tau / T_{\mathrm{s}}}\right)$, which yields a value of $T_{\mathrm{s}}=450 \mathrm{fs}$. Given the simulation results above, it is clearly difficult to define and deconvolve a response function from the $\tau_{\mathrm{s}}$ curve, and attempts to fit the $\tau_{\mathrm{s}}$ curve including convolution with a Gaussian response degraded the quality of the fit. In comparing this value of $T_{\mathrm{s}}$ with the fitted Gaussian response for $N_{\mathrm{ex}}$, one should consider the half width at half maximum of $T_{\mathrm{r}} / 2=210 \mathrm{fs}$. Still, this uncorrected value of $T_{\mathrm{s}}$ is close to $T_{\mathrm{s}}=500 \mathrm{fs}$ reported in Ref. [6] (i.e., for a lower density, $N_{\mathrm{ex}}=3 \times 10^{15} \mathrm{~cm}^{-3}$ ) and quite likely corresponds to subsequent energy relaxation of the photoexcited carriers.

\section{CONCLUSIONS}

The use of THz-MIR spectroscopy with a detailed quantitative analysis has yielded a more comprehensive estimate of the Drude scattering time of charge-carrier plasmas in undoped $\mathrm{Si}$ and its dependence on $N_{\text {ex }}$. The momentum relaxation time of the thermalized plasma ranges from $\sim 200 \mathrm{fs}$ at low electron-hole densities to $\sim 20$ fs for $N_{\text {ex }} \sim 10^{19} \mathrm{~cm}^{-3}$ at room temperature. The direct comparison with theoretical predictions provides mutual support for both and supports the mechanism of scattering rate saturation due to phasespace restrictions at high density. A review of the literature indicates that there are still open issues to address, both experimentally and theoretically, particularly (i) the effect of excitation energy and temperature (in order to account for the differences between our results and those from, e.g., Ref. [7]), (ii) the magnitude of $\tau_{\mathrm{s}}$ at very high densities (where either a revision of the experimental results or additional physical scattering mechanisms would be needed to reconcile the reported values $\tau_{\mathrm{s}} \sim 1 \mathrm{fs}$ ), and (iii) the precise subpicosecond plasma dynamics. In the latter case, state-of-the-art theoretical methods to describe the nonequilibrium dynamics [36] are required. Our ongoing THz-MIR studies with improved time resolution are aimed at providing an experimental test for such theoretical predictions. The results from auxiliary 1D FDTD simulations revealed important aspects for conducting a reliable analysis of time-resolved spectra and demonstrated that the spatiotemporal propagation effects must be taken into account even in a reflection geometry.

\section{ACKNOWLEDGMENTS}

The authors gratefully acknowledge financial support from the Deutsche Forschungsgemeinschaft (DFG) for this research work. 
[1] M. van Exter and D. Grischkowsky, Phys. Rev. B 41, 12140 (1990).

[2] R. A. Soref and B. R. Bennett, IEEE J. Quantum Electron. 23, 123 (1987).

[3] T. T. Mnatsakanov, M. E. Levinshtein, A. G. Tandoev, S. N. Yurkov, and J. W. Palmou, J. Appl. Phys. 97, 103715 (2005).

[4] P. Lorazo, L. J. Lewis, and M. Meunier, Proc. SPIE 3935, 66 (2000).

[5] F. Baffigi, G. Cristoforetti, L. Fulgentini, A. Giulietti, P. Koester, L. Labate, and L. A. Gizzi, Phys. Plasmas 21, 072108 (2014).

[6] J. Zielbauer and M. Wegener, Appl. Phys. Lett. 68, 1223 (1996).

[7] E. Hendry, M. Koeberg, J. Pijpers, and M. Bonn, Phys. Rev. B 75, 233202 (2007).

[8] M. Nagai and M. Kuwata-Gonokami, J. Phys. Soc. Jpn. 71, 2276 (2002).

[9] C. V. Shank, R. Yen, and C. Hirlimann, Phys. Rev. Lett. 50, 454 (1983).

[10] D. Hulin, M. Combescot, J. Bok, A. Migus, J. Y. Vinet, and A. Antonetti, Phys. Rev. Lett. 52, 1998 (1984).

[11] K. Sokolowski-Tinten, J. Bialkowski, and D. von der Linde, Phys. Rev. B 51, 14186 (1995).

[12] K. Sokolowski-Tinten and D. von de Linde, Phys. Rev. B 61, 2643 (2000).

[13] A. J. Sabbah and D. M. Riffe, Phys. Rev. B 66, 165217 (2002).

[14] T. Ichibayashi and K. Tanimura, Phys. Rev. Lett. 102, 087403 (2009).

[15] M. D. Thomson, S. M. Tzanova, and H. G. Roskos, Phys. Rev. B 87, 085203 (2013).

[16] F. Meng, M. D. Thomson, and H. G. Roskos, Phys. Rev. B 90, 155207 (2014).

[17] M. Combescot, Solid State Commun. 62, 587 (1987).

[18] M. Combescot and R. Combescot, Phys. Rev. B 35, 7986 (1987).
[19] B. E. Sernelius, Phys. Rev. B 40, 12438 (1989).

[20] B. E. Sernelius, Phys. Rev. B 36, 1080 (1987).

[21] B. E. Sernelius, Phys. Rev. B 43, 7136 (1991).

[22] M. D. Thomson, V. Blank, and H. G. Roskos, Opt. Express 18, 23173 (2010).

[23] V. Blank, M. D. Thomson, and H. G. Roskos, New. J. Phys. 15, 075023 (2013).

[24] M. D. Thomson, V. Blank, and H. G. Roskos, Eur. Phys. J. Web Conf. 41, 09011 (2013).

[25] Silicon Materials \& Process Control, Tech. Rep. (SEMI International Standard, 2007), http://ams.semi.org/ebusiness/ standards/SEMIStandardDetail.aspx?ProductID $=211 \&$ DownloadID $=1806$.

[26] J. Y. Vinet, M. Combescot, and C. Tanguy, Solid State Commun. 51, 171 (1984).

[27] H. M. Ma, Y. X. Liu, Y. Fei, and F. M. Li, J. Appl. Phys. 65, 5031 (1989).

[28] W. H. Press, S. A. Teukolsky, W. T. Vetterling, and B. P. Flannery, Numerical Recipes (Cambridge University Press, Cambridge, 2007), Chap. 15.

[29] M. Tsubouchi, M. Nagai, and Y. Ohshima, Opt. Lett. 37, 3528 (2012).

[30] H. M. van Driel, Phys. Rev. B 35, 8166 (1987).

[31] T. Sjodin, H. Petek, and H. L. Dai, Phys. Rev. Lett. 81, 5664 (1998).

[32] M. Combescot and J. Bok, Phys. Rev. B 35, 1181 (1987).

[33] E. Pop, R. W. Dutton, and K. E. Goodson, J. Appl. Phys. 96, 4998 (2004).

[34] A. Dargys and J. Kundrotas, Handbook on Physical Properties of Ge, Si, GaAs and InP (Science and Encyclopedia Publishers, Vilnius, Lithuania, 1994).

[35] B. Fischer and K. R. Hofmann, Appl. Phys. Lett. 76, 583 (2000).

[36] R. Binder and S. Koch, Prog. Quantum Electron. 19, 307 (1995). 